\title{
Exploiting graph neural network with one-shot learning for fault diagnosis of rotating machinery (PHM2021)
}

Shuai Yang ( $\square$ jerryyang@ctbu.edu.cn )

Chongqing Technology and Business University https://orcid.org/0000-0001-8015-1578

\section{Xu Chen}

Chongqing Technology and Business University

\section{Yun Bai}

Chongqing Technology and Business University

\section{Ziqiang Pu}

University of Algarve: Universidade do Algarve

\section{Research Article}

Keywords: Rotating machinery, deep learning, fault diagnosis, graph neural network, one-shot learning

Posted Date: March 7th, 2022

DOI: https://doi.org/10.21203/rs.3.rs-1245521/v1

License: (c) (i) This work is licensed under a Creative Commons Attribution 4.0 International License. Read Full License 


\section{Title page}

\section{Exploiting graph neural network with one-shot learning for fault diagnosis of rotating machinery (PHM2021)}

Shuai Yang, born in 1986, is currently an associate professor with Chongqing Technology and Business University, Chongqing, China. He received his $\mathrm{Ph}$. D. degree from University of Ottawa, Canada. His research interests include vibration control and mechanical fault diagnosis.

Tel: +86-23-62933621; E-mail: jerryyang@ ctbu.edu.cn

Xu Chen, born in 1994, is currently a postgraduate student at Chongqing Technology and Business University, China. She received her Bachelor's degree in Dalian ocean university, China, in 2017.

E-mail: chenxu2755473272@163.com

Yun Bai, born in 1985, is currently an associate professor with Chongqing Technology and Business University, Chongqing, China, and also is a postdoctoral researcher at University of Algarve, Portugal. His current research interests include intelligent system modeling and management.

E-mail: yunbai@ctbu.edu.cn

Ziqiang Pu, he is currently a Ph.D. candidate at University of Algarve, Portugal. He received his Bachelor's degree from Chongqing Technology and Business University, China, in 2016. His research interests include mechanical fault diagnosis and intelligent systems E-mail: a66835@ualg.pt

\section{Corresponding author: Shuai Yang E-mail: jerryyang@ctbu.edu.cn}




\title{
Exploiting graph neural network with one-shot learning for fault diagnosis of rotating machinery
}

\author{
Shuai Yang ${ }^{1^{*}} \cdot$ Xu Chen ${ }^{1,2} \cdot$ Yun Bai $^{2} \cdot$ Ziqiang Pu $^{3}$
}

\begin{abstract}
Due to lack of training samples, overfitting is a severe problem in fault diagnosis for mechanical devices, especially for rotating machinery. In this paper, a graph neural network (GNN) method with one-shot learning is proposed for fault diagnosis of rotating machinery. Convolutional Neural Network (CNN) is applied to extract the feature vectors and generate codes for one-shot learning. With adjacency matrix in GNN, the proposed method can achieve fault classification for rotating machinery with small dataset. Rotate vector (RV) reducer of the industrial robot and bearing of the rotating shaft were chosen as experimental subjects. Experimental results show the high accuracy of classification in both experiments with the proposed method. To further verify the efficiency of this method, Siamese Net, Matching Net and SAE+RF were chosen as the comparisons. The results indicate the proposed method outperforms all the selected methods for fault classification in both rotating machineries.
\end{abstract}

Keywords: Rotating machinery, deep learning, fault diagnosis, graph neural network, one-shot learning

\section{Introduction}

Due to the harsh working environment, the failure of rotating machinery is inevitable, which leads to financial losses or even casualties [1,2]. Therefore, the early fault detection and diagnosis of rotating machinery are key strategy to prevent these catastrophic results [3].

Aiming at the problem of fault diagnosis of rotating machinery, scholars have proposed a large number of deep

Shuai Yang

jerryyang@ctbu.edu.cn

1 National Research Base of Intelligent Manufacturing Service, Chongqing Technology and Business University, Chongqing 400067, China

2 School of Management Science and Engineering, Chongqing Technology and Business University, Chongqing 400067, China

Institute of Engineering, University of Algarve, Faro, Portugal learning feature extraction methods, which greatly promoted the development of mechanical fault diagnosis. As a typical method of deep learning, $\mathrm{CNN}$ has made great progress in the fields of image classification, target detection and image semantic segmentation in recent years [4, 8]. Chen et al. [9] fused the horizontal and vertical vibration data into a two-dimensional matrix and proposed a deep CNN to identify the health status of the gearbox. By considering the characteristics of industrial signals, Yang et al. [10] converted multi-source vibration signals into a two-dimensional matrix and proposed a CNN-based fault diagnosis method for reciprocating compressors. Zhao et al. [11] monitored and diagnosed tool wear through the combination of CNN and long short term memory (LSTM). Through the above method, a better fault diagnosis result was finally obtained. Wang et al. [12] proposed a conversion method to convert vibration signals from multiple sensors to images. This method can obtain feature maps of different fault types 2 without tedious parameter adjustments.

In recent years, as a popular research direction of artificial intelligence. GAN has been wildly adopted for mechanical fault diagnosis [13, 16]. Lee et al. applied GAN network to the fault detection of asynchronous motors to solve the problem of data imbalance caused by small amount of data [17]. Plakias et al. proposed a multi-dimensional problem single-class fault detection scheme based on unsupervised training of GAN [18]. Wang et al. [19] combined GAN with stack noise reduction automatic encoder to generate planetary gearbox fault data; Mao et al. [20] solved the problem of data imbalance in bearing fault diagnosis by combining GAN and stacked noise reduction automatic encoder. The results show that the fault samples generated by GAN can significantly improve the fault diagnosis accuracy in the case of data imbalance.

As mentioned above, the fault diagnosis of mechanical system with CNN and GAN have archived great progress 
in last several decades [21]. However, for small data of rotating machinery, the network structure design for the characteristics of the signals is missing in both GAN and $\mathrm{CNN}$.

In this paper, a GNN with one-shot learning method is proposed to carry out mechanical fault classification. Experiments were carried out to verify the efficiency of the proposed method. Siamese Net [22], Matching Net [23] and SAE+RF [24] were chosen as the comparisons.

The paper is structured as the follows. Section 2 introduces the theoretical basis and the whole process of the proposed method. Section 3 evaluates the proposed approach for rotating machinery fault diagnosis with experiments. Section 4 provides results with a discussion on the case. Conclusions are drawn in Section 5.

\section{Methodology}

\subsection{Short-Time Fourier Transform (STFT)}

For non-stationary signals, other than frequency-domain signals, the time domain signals also need to be analyzed. In this paper, the STFT is chosen for transforming one-dimensional data into two-dimensional $[25,26]$. The specific principle of STFT is listed as the follows.

1) Move the window to the starting point of the signal. At this time, the center position of the window function is at $\mathrm{t}=\beth_{-} 0$, the signal is windowed:

$$
y(t)=x(t) * w\left(t-\beth_{0}\right)
$$

Where $t$ is the time translation parameter, $x(t)$ is the original signal. $w\left(t-\beth_{0}\right)$ is the sequence of real window functions. The intercepted signal $y(t)$ can be obtained by multiplying the window function and the original signal. The intercepted signal $y(t)$ is the signal of execution time corresponding to $t$.

2) By the Fourier transform, equation (1) can be rewritten as:

$$
X(\omega)=\mathcal{F}(x(t))=\int_{\infty}^{+\infty} x(t) * w\left(t-\beth_{0}\right) e^{-j w t} d t
$$

$X(\omega)$ is the spectral distribution of the first segmented sequence. In practical applications, since the signal is a sequence of discrete points, the spectral sequence $\mathrm{X}[N]$ can be obtained.

3 ) For the convenience of expression, the function $S(\omega, \beth)$ is defined:

$$
\begin{gathered}
S(\omega, \beth)=\mathcal{F}(x(t) * w(t-\beth))=\int_{\infty}^{+\infty} x(t) * w(t- \\
\left.\beth_{0}\right) e^{-j w t} d t
\end{gathered}
$$

The function $S(\omega, \beth)$ represents the spectral result $X(\omega)$ after transformation of the original function when the window function center is $\beth_{-} O$.

4 ) the spectral energy relationship of time can be determined by:

$$
\begin{gathered}
\delta_{S P}(\omega, \beth)=\|S(\omega, \beth)\|^{2}=\| \int_{\infty}^{+\infty} x(t) * w(t- \\
\left.\beth_{0}\right) e^{-j w t} d t \|^{2}
\end{gathered}
$$

\subsection{Using CNN to extract feature vectors}

$\mathrm{CNN}$ is a feedforward neural network with deep structure. There are three important concepts to know about the convolution process: local perception, parameter sharing and pooling.

Let the input of convolutional neural network be the original image $P . F_{i}$ represents the convolution layer, so the generation process of $F_{i}$ can be described as:

$$
F_{i}=f\left(F_{i-1} \otimes W_{i}+b_{i}\right)
$$

Where $W_{i}$ represents the weight vector of the convolution kernel at layer $i, b_{i}$ represents the offset vector at layer $i . \otimes$ represents to use the convolution kernel to perform convolution operation on the feature map of layer $i-1$. Finally, the feature map $F_{i}$ of the layer $i$ is obtained through the nonlinear excitation function $f$.

The pool layer usually follows the convolution layer and samples the feature map according to certain down sampling rules. Assuming $S_{i}$ is the pool layer, the operation of pool can be described as:

$$
S_{i}=\operatorname{sub}_{\text {sampling }}\left(S_{i-1}\right)
$$

Where $s u b_{\text {sampling }}$ is a downsampling function, including maximum pooling, average pooling, or random pooling.

Feature extraction is being performed in every $F_{i}$, where the convolutional layer obtains the main features through convolution operation and nonlinear activation function. In the paper, the vertices of the graph are represented as a [image-embedding, label] vector, which the image-embedding is the feature extracted by the image through a convolutional neural network. The convolutional layer and the pooling layer can extract image features and determine the parameters of the convolutional kernel through back propagation to obtain the final features, which is a rough process of CNN feature extraction [27, 29]. 


\subsection{GNN learned with one-shot learning}

The key concept for small sample learning method is learning the relationship between training sets and query sets by itself. GNN is a graph model formed by nodes and edges [30]. It maps every image of the training data to a vertex on the graph. Through training, it obtains the adjacency matrix between the vertices in the graph and uses it to classify and infer. This method can effectively represent the complex relationship between data. Therefore, GNN is applied to one-shot learning to meet the requirements of processing structural information between data in the process of one-shot learning.

In this paper, each node represents an image. The weight of each edge represents the relationship between the two images (distance or similarity) [31, 32]. Specific weight calculation process:

$$
\tilde{A}_{i, j}^{(k)}=\varphi_{\widetilde{\theta}}\left(x_{i}^{(k)}, x_{j}^{(k)}\right)=M L P_{\widetilde{\theta}}\left(a b s\left(x_{i}^{(k)}-x_{j}^{(k)}\right)\right)
$$

Where $x^{k}$ is received as input of a GNN layer $G c()$, where $\varphi=M L P$ is a symmetric function parametrized with e.g. a neural network. In this paper, a Multilayer Perceptron stacked is considered after the absolute difference between two vector nodes.

As shown in Figure 1, among $\varphi_{\widetilde{\theta}}()$ input the absolute difference between two nodes, which can be learned by nonlinear combination of the absolute differences between the features of two nodes. Using this architecture, the symmetric distance characteristic is satisfied by construction $\varphi_{\widetilde{\theta}}(a, b)=\varphi_{\widetilde{\theta}}(b, a)$. It is easy to determine its own distance characteristic $\varphi_{\widetilde{\theta}}(a, a)=0$ and output the corresponding weight value.

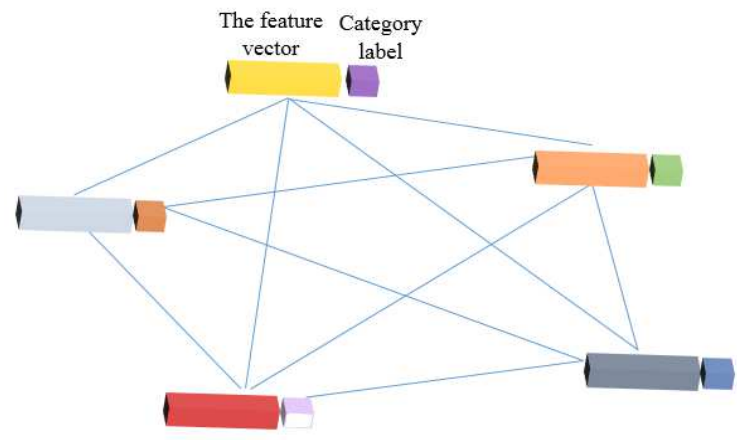

Figure 1 The connection matrix for node connections

The transformations are applied to embedding through GNN. With a fully connected layer and Leakyrelu function, a new embedding can be generated. to produce a new embedding. The new embedding ensures that the sum of weights between each node and all other nodes is 1 . After the adjacency matrix is obtained by the above method, the next layer network can be calculated by graph convolution neural network (GNN) to complete GNN transfer. The calculation process is as follows:

$x_{l}^{(k+1)}=G c\left(x^{(k)}\right)=\rho\left(\sum_{B \in A} B x^{(k)} \theta_{B, l}^{(k)}\right), l d_{1} \ldots d_{k+1}$,

Where $x_{l}^{(k+1)}$ represents the node of the $k+1$ layer network, $l$ represents the length of the eigenvector on the node, $\rho(\cdot)$ represents the nonlinear activation function.

$$
\Theta=\left\{\theta_{1}^{(k)}, \ldots, \theta_{|A|}^{(k)}\right\}_{k}, \theta_{A}^{(k)} \in \mathbb{R}^{d_{k}^{*} d_{k+1}}
$$

represents the set of training parameters. The accumulation symbol indicates that the adjacency matrix B can adopt a variety of calculation methods and add them together. According to this formula, the update rule of the node feature can be obtained.

As shown in Figure 2, due to the denseness of the edges in the graph, depth is simply interpreted as giving the model more expressive power. However, there are only two cases in this article. The first is that the weight of the connection between the node and itself is 1 . The second is that the weight of the connection with other nodes is $\tilde{A}_{i, j}^{(k)}$. In the training process, it is also necessary to change the weight of each layer of the network. The input $V^{(k)}$ and the output of the Gc block are cascaded to generate the lower-level network input $V^{(k+1)}$.

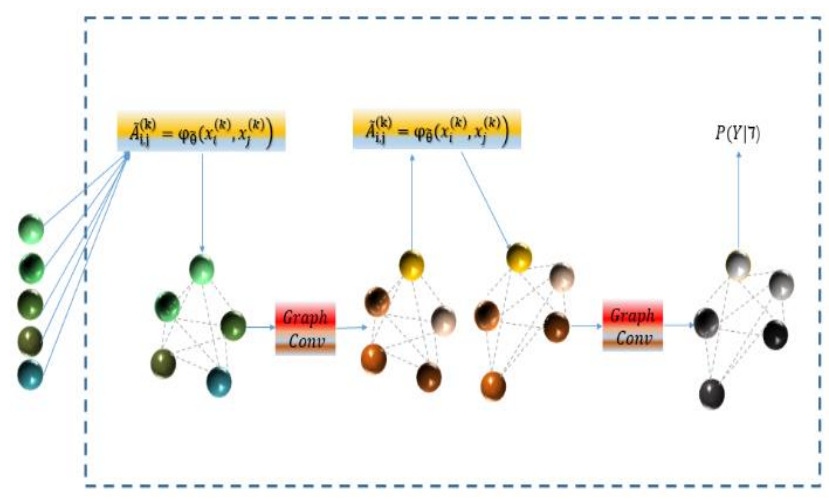

Figure 2. The typical structure of the GNN

The feature of initial point is defined as:

$$
x_{i}^{(0)}=\left(\phi\left(x_{i}\right), h\left(l_{i}\right)\right)
$$

Where $\phi(\cdot)$ is a Convolutional neural network, $h(\cdot)$ represents the translation of the tag into a one-hot vector.

The final loss function is:

$$
\min \frac{1}{L} \sum_{i \leq L} \ell\left(\Phi\left(T_{i} ; \theta\right), Y_{i}\right)+\mathcal{R}(\theta)
$$


Where $T_{i}$ is the $\mathrm{i}$-th time of the task, the targets $Y_{i}$ are associated with image categories of designated images $x_{i}, \ldots x_{j} \in T_{i}$ with no observed label. $\left\{\left(T_{i}, Y_{i}\right)_{i}\right\}_{i \leq L}$ is a training set.

$$
\ell(\Phi(\urcorner ; \theta), Y)=-\sum y_{k} \log P\left(Y_{*}=y_{k} \mid T\right)
$$

Where $\Phi(T ; \theta)=P(Y \mid T)$, the predicted label is obtained through Maximum likelihood estimation.

The combination of graph neural network and one-shot learning in meta-learning can be applied to fault diagnosis of rotating machinery. The details are illustrated in Figure 3 and summarized below.

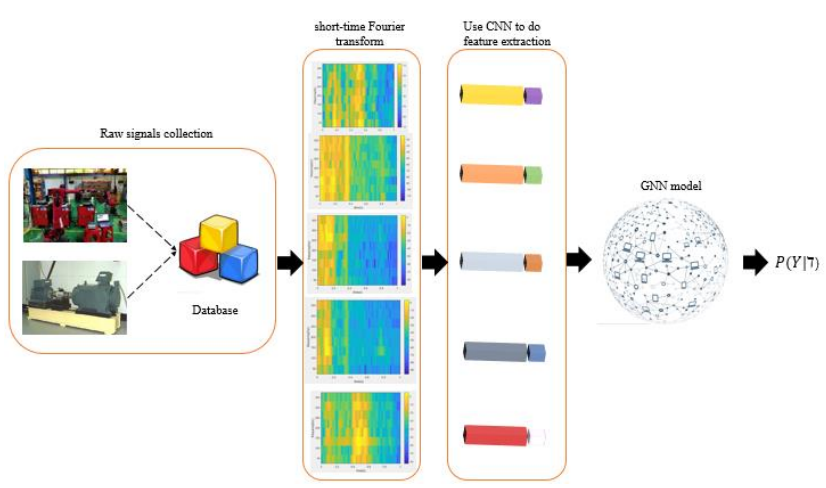

Figure 3. The working flowchart of the proposed method

Step 1. Collect signals from industrial robot acceleration sensors and public dataset CWRU under health and failure conditions, reflecting the actual situation from multiple dimensions.

Step 2. The fault state signal of one-dimensional rotating machinery is transformed into two-dimensional picture by short-time Fourier transform.

Step 3. Use CNN to extract feature vectors.

Step 4. Input the feature vector into the graph neural network and apply the graph neural network to the learning of a few samples.

Step 5. Obtain the predicted results for fault diagnosis and classification.

\section{Experiments}

\subsection{Datasets}

In order to verify the effectiveness of this method, experiments with different rotation devices were carried out in this paper. The first one is the RV reducer of industrial robots, the other one is the benchmark rolling bearing dataset made by Case Western Reserve University (CWRU).

For RV reducer, the BRTIRUS1510A 6-DOF industrial robot of Guangdong Bronte Intelligent Equipment Incorporated Company is chosen. This robot equips with six joints, six servo motors and six reducers. All the experiments were carried out on J1 axis reducer, which is one of the most important and frequently used components. Under different fault conditions, the data were collected by using PCB acceleration sensor. In the experiment, the sampling frequency was set to $100 \mathrm{~Hz}$. To be more accurate, robot movement conditions were set with different speeds under 3 different loads, i.e., $0 \mathrm{~kg}, 4.8 \mathrm{~kg}$ and $9.6 \mathrm{~kg}$ loads with $600 \mathrm{r} / \mathrm{min}, 1500 \mathrm{r} / \mathrm{min}$ and $2400 \mathrm{r} / \mathrm{min}$ speed, respectively. Under predefined conditions (one normal, four faults), the same trajectory was used to control the motion of the robot. In this way, experimental datasets with healthy and different faulty modes were established. The faulty modes used in this article include the following five types: (1) full health medium speed intermediate load $(4.8 \mathrm{~kg})$, (2) Moderate pitting and medium speed intermediate load of sun gear A (4.8kg), (3) Medium speed intermediate load of sun gear A full broken teeth $(4.8 \mathrm{~kg})$, (4) Medium speed intermediate load of planetary gear A WEDM groove (4.8kg), (5) Medium speed intermediate load of planetary gear A with broken teeth $(4.8 \mathrm{~kg})$. Each type of industrial robot generated an image with 20000 data points and collected 1000 images, a total of $1000 * 5$ images. 7000 pictures were used as train data, 1500 pictures were used as validation data. The rest 1500 pictures were used as test data.

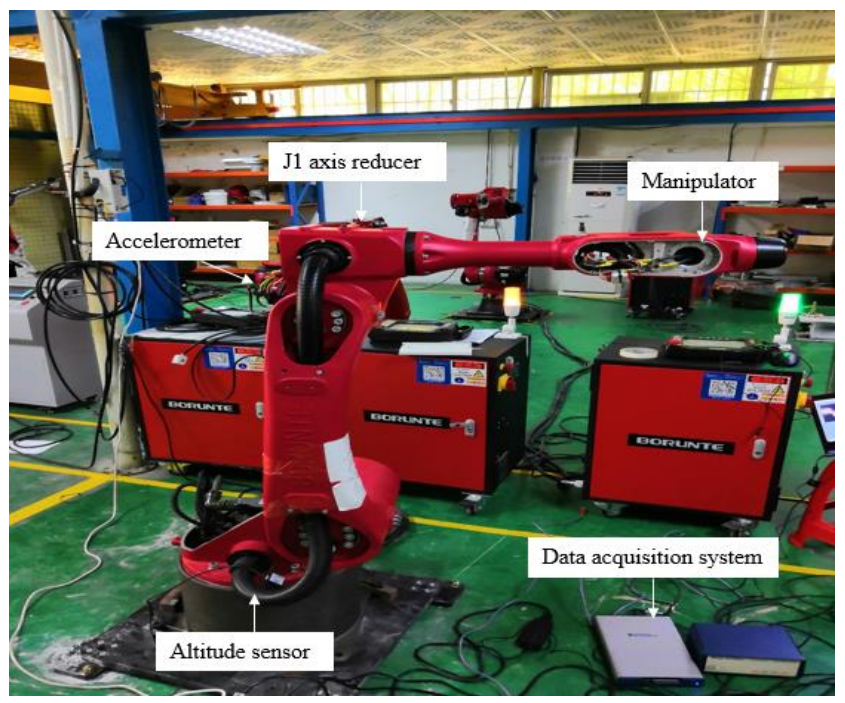

Figure 4. The experimental setup of industrial robot

For the other experiments, motor bearing fault data signals of CWRU were selected. The CWRU dataset was 
obtained using accelerometers to collect vibration data from a test-rig consists of a 2-HP motor, a torque transducer and a dynamometer. Two sampling frequencies (12 kHz and $48 \mathrm{kHz}$ ) were set in the experiments to collect data from four types of condition patterns, i.e., (1) Normal Baseline Data; (2) 12k Drive End Bearing Fault Data; (3) 48k Drive End Bearing Fault Data; (4) 12k Fan End Bearing Fault Data; In addition, since ORF is stationary fault, different fault placements located at 3 o'clock (ORF-3), 6 o'clock (ORF-6) and 12 o'clock (ORF-12) were also considered. Four fault diameters (i.e., 0.007 inches, 0.014 inches, 0.021 inches and 0.028 inches) were pre-planted for IRF, RBF, ORF-3, ORF-6 and ORF-12, separately. In total, data were acquired from 80 condition categories by combining different sampling frequencies, condition patterns, fault diameters and motor speeds. We randomly selected 55 classes to form the train dataset, where each class has 300 samples. The remaining classes were randomly divided into a validation dataset (10 classes) and a test dataset (15 classes). Each type of motor bearing data generated a picture with 480 data points and collected 300 images for a total of $300 * 80$ pictures.

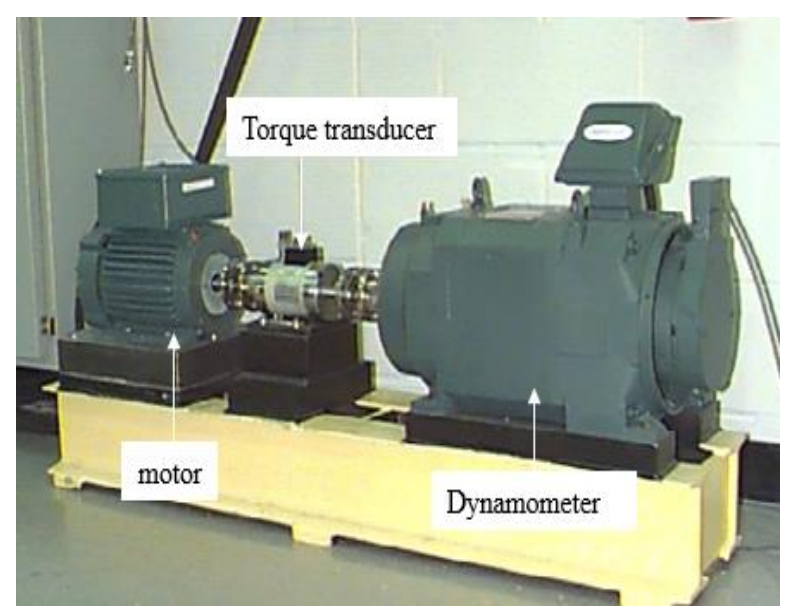

Figure 5 The experimental setup of bearing (CWRU)

\subsection{Workflow}

In this paper, the one-dimensional signals of rotating machinery are transformed into two-dimensional pictures by STFT. After STFT processing, the time domain diagram is changed into the frequency domain diagram As shown in Fig 6, corresponding to the discrete scene, it is a two-dimensional matrix $S[\omega, \beth]$. Each column represents the sequence of the result after the Fourier transform of the obtained segment by windowing the signal at different positions. After the FFT of the first segment is completed, the window function will be moved to $\beth_{1}$. The distance of the window moves is called hop size. Generally, the moving distance is smaller than the width of the window to ensure a certain overlap between the two near windows, which is called overlap. Spectrum results for all segments from $\beth_{0} \sim \beth_{N}$ were obtained by repeating the above operation, sliding window, FFT, which is the result of STFT transformation. The one-dimensional data can be converted into the picture form of the two-dimensional data by the above method. All raw data was converted into image data as shown in Section 2.1. Image data was divided into two parts. Output data was formed by stacking information such as image, label, one-hot corresponding to label and its class in the dataset.

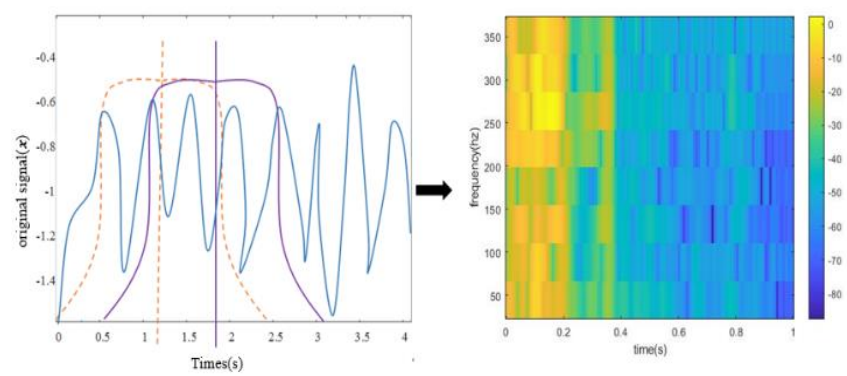

Figure 6 Data process by short-time Fourier transform

Feature vectors are extracted by CNN. The convolutional layer and the pooling layer can extract image features and determine the parameters of the convolutional kernel through back propagation to obtain the final features. Through the initial value setting of $\mathrm{CNN}$, each image was finally processed into a 64-dimensional feature vector. The image was fed into the neural network using the method in Section 2.2, with Leakyrelu as the activation function and the cross-entropy loss function as the loss measure, using the Adam optimizer with an initial learning rate of 0.001 .

The feature vector is input into GNN and applied to the one-time sample learning of GNN. In the experiment, $\mathrm{N}$-way K-shot was used as the evaluation index. The model was evaluated by performing 1-shot, 5-way experiments on the dataset. For each one-shot task, we sampled 5 classes from the dataset and 1 random sample from each class. An additional sample was selected from the five categories for classification. The classification accuracy was obtained by performing 5-way 1 -shot and 5-way 5-shot to verify the superiority of the proposed algorithm. Finally, the prediction results are obtained for classification of fault diagnosis of rotating machinery.

\section{Results and discussion}

In experiment 1, 5000 samples were collected for all categories. To illustrate, the signal corresponding to the 
health status of the industrial robot was plotted in Figure 7

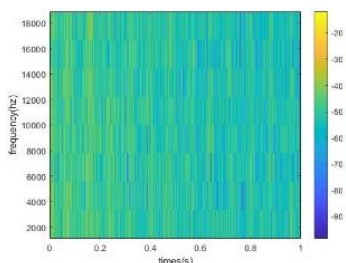

(a)

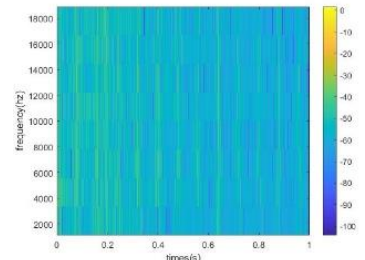

(b)

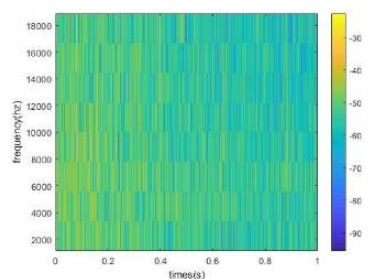

(c)

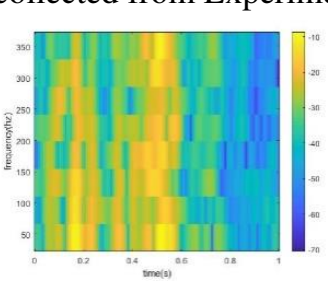

(a)

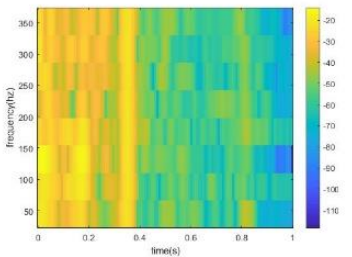

(b)

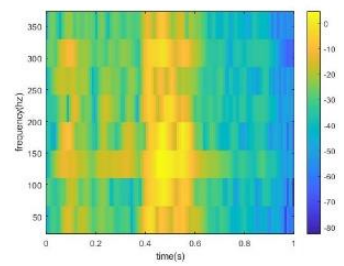

(c)

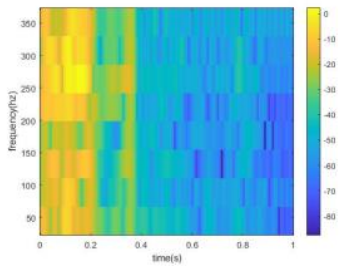

(d)

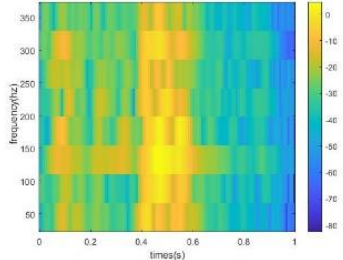

(e) (d)

(e)

Figure 7 Signals acquired from RV reducer of industrial robot. (a) Healthy state; (b) Pitting in Sun gear A; (c) Broken tooth in Sun gear A; (d) cutting groove in Planetary gear A and (E) Cracking in Planetary gear A

5-way 1-shot, 2-shot, 3-shot, 4-shot and 5-shot were performed on industrial robot datasets in Figure 8. Since each category of 5-way 5-shot has five more data samples than 5-way 1-shot, the accuracy of 5-way 5-shot (Accuracy of $96.89 \%$ ) is higher than that of 5-way 1-shot (Accuracy of $96.56 \%$ ).

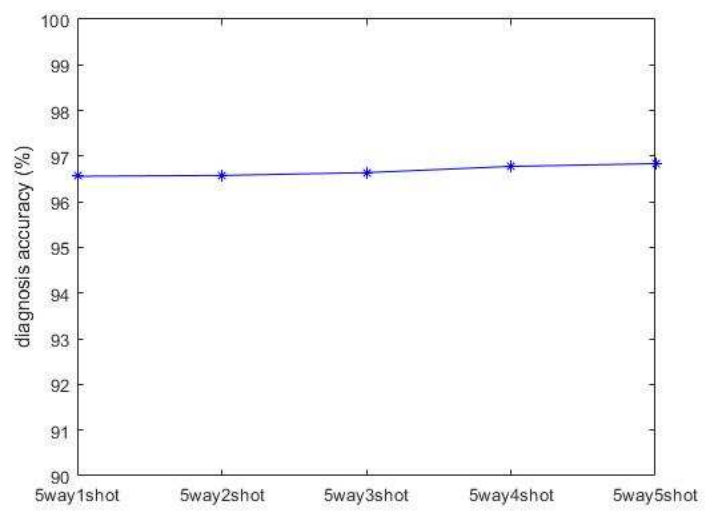

Figure 8 Classification accuracy for RV reducer of industrial robot

A total of 24,000 samples of CWRU datasets were
Figure 9 Signals acquired from bearings of CWRU: (a) Healthy state; (b) Drive end bearing fault data of inner race; (c) Drive end bearing fault data of ball; (d) Fan end bearing fault data of inner race and (E) Fan end bearing fault data of ball

5-way 1-shot, 2-shot, 3-shot, 4-shot and 5-shot were performed on CWRU datasets in Figure 8.The accuracy of 5-way 5-shot (Accuracy of 97.89\%) is higher than that of 5-way 1-shot (Accuracy of $95.42 \%$ )

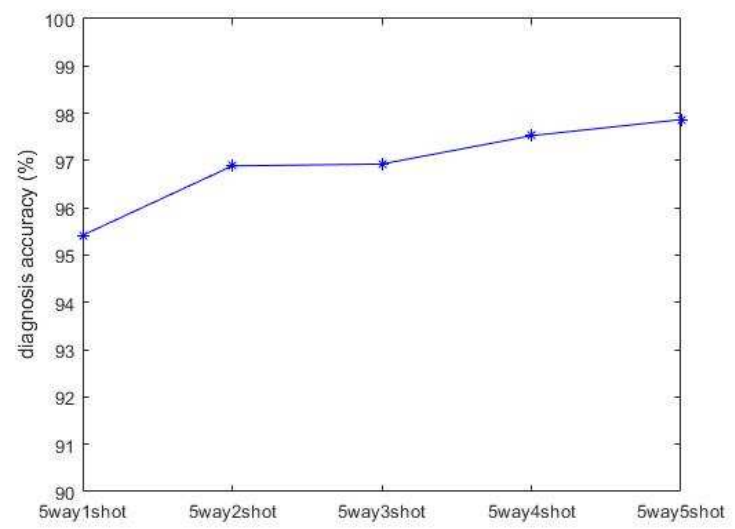

Figure 10 Classification accuracy for bearing of CWRU

For few-shot fault diagnosis problems, the number of shots (i.e. the value of $\mathrm{K}$ ) diametrically reflects the 
intricacy of the problem structure. In fact, the smaller the value of $K$, the harder the learning task is [33]. The accuracy of 1-shot and 5-shot classification tasks has been significantly improved. Because the model can learn more representative features from more labeled samples for classification. At the same time, it also proves that the algorithm enhances the measurement relationship between node edges by optimizing the correlation between graph nodes and samples. It can obtain excellent classification accuracy by using label propagation to predict the unknown sample tags.

For comparisons, different approaches were used for fault conditions with the same dataset, which are Siamese Net, Matching Net, SAE+RF $[34,36]$. Among them, Siamese Net is recognized as a simple and effective method, which is widely used in computer science, finance, medicine and other fields. Matching Network can combine the attention structure and the memory network to build a fast-learning network. SAE is an unsupervised learning algorithm, which has fewer parameters and is not easy to cause over fitting compared with traditional artificial neural network; RF is a very effective and competitive classification solution on overfitting problems. It can evaluate the importance of each feature in classification problems. Therefore, Siamese Net, Matching Net and SAE $+\mathrm{RF}$ were used for comparison.

Table 1 shows the comparison of the different models. Obviously, GNN had the highest classification rate each time ( $96.43 \%$ on average). The accuracy of Siamese Net is $81.54 \%$.

The accuracy of SAE+RF $t$ is $83.39 \%$. The performance of Matching Net is the worst, only $81.36 \%$ in average. It indicates that GNN is the best classification method for industrial robot fault diagnosis.

As shown in Table 2, Similarly, Siamese Net, Matching Net, SAE+RF and GNN are also applied to CWRU dataset. The absolute advantage of GNN can also be found.

Table 1 The classification results of different method with industrial robot dataset

\begin{tabular}{ccccccc}
\hline \hline \multirow{2}{*}{ Model } & \multicolumn{5}{c}{ Accuracy of each test (\%) } & \multirow{2}{*}{ Mean (\%) } \\
\cline { 2 - 5 } & $\mathbf{1}$ & $\mathbf{2}$ & $\mathbf{3}$ & $\mathbf{4}$ & $\mathbf{5}$ & \\
\hline Siamese Net & 80.20 & 83.25 & 81.20 & 82.05 & 81.00 & 81.54 \\
Matching Net & 82.30 & 79.20 & 83.30 & 81.70 & 80.30 & 81.36 \\
SAE+RF & 83.57 & 86.21 & 81.54 & 82.76 & 83.36 & 83.49 \\
GNN & $\mathbf{9 6 . 5 6}$ & $\mathbf{9 6 . 6 3}$ & $\mathbf{9 6 . 2 6}$ & $\mathbf{9 5 . 9 1}$ & $\mathbf{9 6 . 8 1}$ & $\mathbf{9 6 . 4 3}$ \\
\hline
\end{tabular}

Table 2. The classification results of different method with CWRU dataset

\begin{tabular}{ccccccc}
\hline \hline & \multicolumn{7}{c}{ Accuracy of each test (\%) } \\
Model & $\mathbf{1}$ & $\mathbf{2}$ & $\mathbf{3}$ & $\mathbf{4}$ & $\mathbf{5}$ & Mean (\%) \\
\cline { 2 - 5 } & 86.40 & 87.80 & 89.20 & 87.20 & 88.00 & 87.72 \\
Siamese Net & 85.00 & 84.73 & 83.51 & 87.12 & 86.35 & 85.34 \\
Matching Net & 81.23 & 80.22 & 82.25 & 82.13 & 80.53 & 81.27 \\
SAE+RF & $\mathbf{9 5 . 4 2}$ & $\mathbf{9 5 . 3 9}$ & $\mathbf{9 6 . 2 9}$ & $\mathbf{9 6 . 2 7}$ & $\mathbf{9 6 . 5 4}$ & $\mathbf{9 5 . 9 8}$ \\
\hline
\end{tabular}


For better validation, a Box plot is applied for comparisons. It is a statistical plot used to show the dispersion of a set of data, which can reflect the difference in accuracy. The more concentrated the Box plot, the smaller the difference in accuracy. In this paper, a Box plot can be used to reflect the distribution characteristics of the original data of rotating machinery failure and compare the distribution characteristics of multiple groups of data. As it can be seen in Figure 11, the distribution of GNN algorithm is the densest, followed by the SAE+RF distribution. The distribution results of Siamese Net and Matching are much worse than the other two. GNN is better than that of SAE+RF, Matching Net and Siamese Net in the density or fault accuracy of the three.

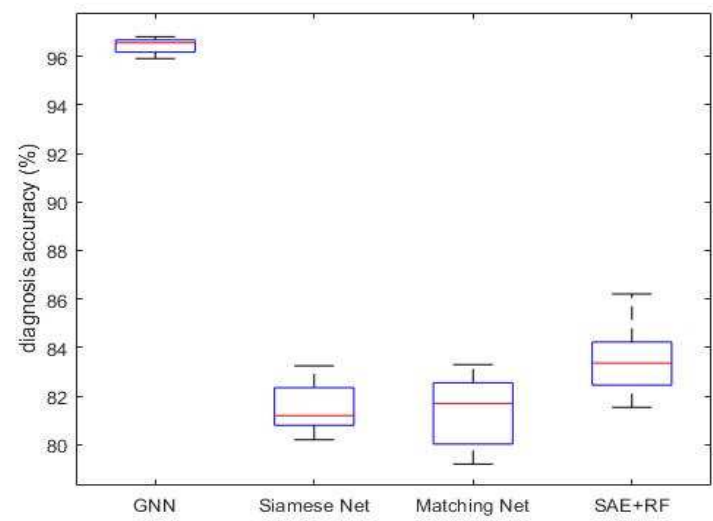

Figure 11 Box-plot of fault diagnosis results by different algorithms of industrial robot dataset

As shown in Figure 12, the distribution of GNN algorithm is the densest. So GNN is the best choice among the four.

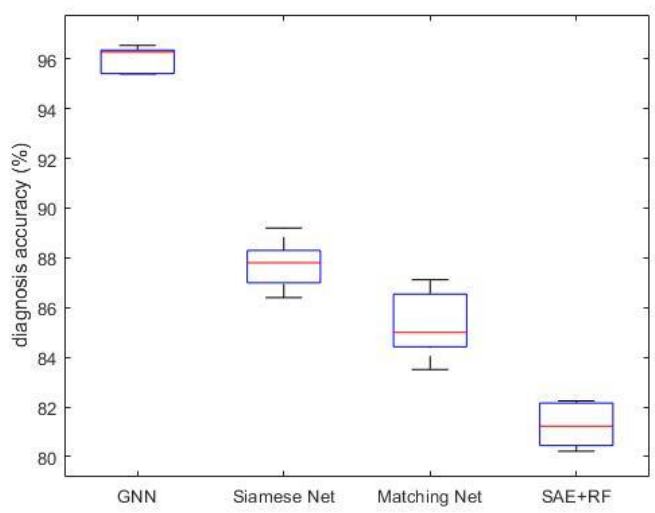

Figure 12 Box-plot of fault diagnosis results by different algorithms of CWRU dataset

GNN is the learning of graphs, which is different from the learning of data itself. It is the learning of graph structure expressed by datasets. The concept of GNN is more indirect, more complex and difficult to understand than ordinary machine learning.

\section{Conclusions and future works}

A classification method for fault diagnosis of rotating machinery is proposed in this paper. By acquiring acceleration signals and motor bearing signals, the short-time Fourier transform (STFT) is used to generate two-dimensional images. The feature vectors are extracted through the convolution neural network (CNN), which are input to the GNN network using the Adjacency Matrix between vertices in its graph for classification and inference. Experiments were carried out to verify the efficiency of the proposed method. Siamese Net, Matching Net and SAE+RF are chosen as the comparisons, the results indicate the proposed method out-performs all the selected methods. The overall accuracy of the proposed method can reach to $95.98 \%$.

In this paper, only the rotating machinery is chosen as the researching object for the proposed method. The fault diagnosis efficiency of this method for other mechanical devices with small sample still need to be verified. Therefore, transfer learning will be applied to the proposed method in our future research. Through transfer learning, the proposed GNN fault diagnosis model for rotating machinery can be applied to other mechanical devices directly.

\section{Declaration}

\section{Acknowledgements}

The authors sincerely thanks to all the editors and reviewers for their efforts on this manuscript.

\section{Funding}

This research is funded by National Natural Science Foundation of China (51905058), Natural Science Foundation of Chongqing (cstc2020jcyj-msxmX0182), Science and Technology Research Program of Chongqing Municipal Education Commission (KJZD-K202100804), the Venture \& Innovation Support Program for Chongqing Overseas Returnees (cx2021075), Key Research Platform Project of Chongqing Technology and Business University (ZDPTTD201918), Research Start-Up Funds of Chongqing Technology and Business University (1856018).

\section{Availability of data and materials}


The second bearing vibration dataset is publicly available from Case Western Reserve University (CWRU) Bearing Data Center.

(http://csegroup.case.edu/bearingdatacenter/home)

\section{Authors' contributions}

The author' contributions are as follows: Shuai Yang was in charge of the whole experiments; Xu Chen wrote the manuscript; Yun bai and Ziqiang $\mathrm{Pu}$ was in charge of programming.

\section{Competing interests}

The authors declare no competing financial interests.

\section{Consent for publication}

Not applicable

\section{Ethics approval and consent to participate}

Not applicable

\section{References}

[1] WEI LI, YIKUN WU . Analysis on the Present Situation and Development Strategy of Industrial Robot Industry in China [J] Gas turbine and accessories, 2018(3): 260-261.

[2] Liao, Y., Huang, R., Li, J. et al. Correction to: Dynamic Distribution Adaptation Based Transfer Network for Cross Domain Bearing Fault Diagnosis. Chin. J. Mech. Eng. 34, 73 (2021). https://doi.org/10.1186/s10033-021-00592-1.

[3] Bao Cai,Qiuyu Shi.Virtual Simulation Development of ABB Industrial Robot Technology[J].Academic Journal of Engineering and Technology Science,2019,2(4).

[4] Pan, H., He, X., Tang S., Meng, F. (2018). An improved bearing fault diagnosis method using one-dimensional CNN and LSTM. Strojniški vestnik - Journal of Mechanical Engineering, vol. 64, no. 7-8, p. 443-452.

[5] HOANG D T, KANG H J. Rolling element bearing fault diagnosis using convolutional neural network and vibration image [J]. Cognitive Systems Research, 2019, 53: 42-50.

[6] XU G, LIU M, JIANG Z, et al. Bearing fault diagnosis method based on deep convolutional neural network and random forest ensemble learning[J]. Sensors, 2019, 19(5): 1088.

[7] EREN L, INCE T, KIRANVAZ S. A generic intelligent bearing fault diagnosis system using compact adaptive 1D CNN classifier [J]. Journal of Signal Processing Systems, 2019, 91(2): 179-189.

[8] Lu, K., Gu, J.X., Fan, H. et al. Acoustics Based Monitoring and Diagnostics for the Progressive Deterioration of Helical Gearboxes. Chin. J. Mech. Eng. 34, $82 \quad$ (2021) https://doi.org/10.1186/s10033-021-00603-1.

[9] CHEN H, HU N, CHENG Z, et al. A deep convolutional neural network based fusion method of two-direction vibration signal data for health state identification of planetary gearboxes [J]. Measurement, 2019, 146: 268-278.

[10] YANG H, ZHANG J, CHEN L, et al. Fault diagnosis of reciprocating compressor based on convolutional neural networks with multisource raw vibration signals[J]. Mathematical Problems in Engineering, 2019.
[11] Rui Z, Ruqiang Y, Jinjiang W, et al. Learning to Monitor Machine Health with Convolutional Bi-Directional LSTM Networks[J]. Sensors, 2017, 17(2):273.

[12] WANG H, LI S, SONG L, et al. A novel convolutional neural network based fault recognition method via image fusion of multi-vibration-signals[J]. Computers in Industry, 2019, 105: 182-190.

[13] Ramponi G , Protopapas P , Brambilla M , et al. T-CGAN: Conditional Generative Adversarial Network for Data Augmentation in Noisy Time Serieswith Irregular Sampling[J]. 2018.

[14] Kim T, Cha M, Kim H, et al. Learning to discover cross-domain relations with generative adversarial networks[C]. in: Proceedings of the 34th International Conference on Machine Learning-Volume 70. JMLR. org, 2017: 1857-1865.

[15] ZHAO R, YAN R, CHEN Z, et al. Deep learning and its applications to machine health monitoring[J]. Mechanical Systems and Signal Processing, 2019, 115: 213-237.

[16] LIU R, MENG G, YANG B, et al. Dislocated time series convolutional neural architecture: An intelligent fault diagnosis approach for electric machine $[\mathrm{J}]$. IEEE Transactions on Industrial Informatics, 2017, 13 ( 3 ) :1310-1320.

[17] Lee Y O, Jo J, Hwang J. Application of deep neural network and generative adversarial network to industrial maintenance: A case study of induction motor fault detection[C]// 2017 IEEE International Conference on Big Data (Big Data). IEEE, 2018. 3248 - 3253 .

[18] Plakias S, Boutalis Y S. Exploiting the generative adversarial framework for one-class multi-dimensional fault detection[J]. Neurocomputing, 2019,332:396-405.

[19] WANG Z, WANG J, WANG Y. An intelligent diagnosis scheme based on generative adversarial learning deep neural networks and its application to planetary gearbox fault pattern recognition [J] . Neurocomputing, 2018, 310: 213-222.

[20] MAO W, LIU Y, DING L, et al. Imbalanced fault diagnosis of rolling bearing based on generative adversarial network: A comparative study [J]. IEEE Access, 2019, 7: 9515-9530.

[21] TANG S, SHEN C, WANG D, et al. Adaptive deep feature learning network with Nesterov momentum and its application to rotating machinery fault diagnosis $[\mathrm{J}]$. Neurocomputing, 2018, 305: 1-14.

[22] Wang J, Fang Z, Lang N, et al. A multi-resolution approach for spinal metastasis detection using deep Siamese neural networks [J]. Computers in Biology and Medicine, 2017, 84: 137-146.

[23] Vinyals O, Blundell C, Lillicrap T, et al. Matching networks for one shot learning[C] //Proceedings of the Annual Conference on Neural Information Processing System. Cambridge:MIT Press, 2016: 3630-3638

[24] Li Qing-qing, Hou Rui-chun, Ding Xiangqian. Fault diagnosis of rolling bearing based on improved stacking self encoder [J].Computer engi-neering and design, 2019, 40 (7) : 2064-2070.

[25] Eddine C D, Noura M. H fault estimation for nonlinear discrete time-delay system with actuator and sensor faults using nonlinear ts fuzzy models[C]//2017 5th International Conference on Electrical Engineering-Boumerdes (ICEE-B). IEEE, 2017: 1-6.Imranul Islam S , Lim C C , Shi P . Robust fault detection of T-S fuzzy systems with time-delay using fuzzy functional observer[J].

[26] Gao Huizhong, Liang Lin, Chen Xiaoguang,et al. Feature extraction and recognition for rolling element bearing faultutilizing short-time fourier transform and non-negative matrix factorization [J]. Chinese Journal of Mechanical Engineering, 2015, 28: 96-105.

[27] LeCun, Y., Bottou, L., Bengio, Y., Haffner, P. (1998). 
Gradient-based learning applied to document recognition. Proceedings of the IEEE. vol. 86, no. 11, p. 2278-2324.

[28] M. Gori, G. Monfardini, and F. Scarselli, "A new model for learning in graph domains," in Proceedings of the International Joint Conference on Neural Networks, vol. 2. IEEE, 2005, pp. 729-734.

[29] Wang, S., Chen, H. (2019). A novel deep learning method for the classification of power quality disturbances using deep convolutional neural network. Applied Energy, vol. 235, p. 1126-1140,

[30] F. Scarselli, M. Gori, A. C. Tsoi, M. Hagenbuchner, and G. Monfardini, "The graph neural network model," IEEE T ransactions on Neural Networks, vol. 20, no. 1, pp. 61-80, 2009.

[31] Y . Li, D. Tarlow, M. Brockschmidt, and R. Zemel, "Gated graph sequence neural networks," in Proceedings of the International Conference on Learning Representations, 2015.

[32] Oriol Vinyals, Charles Blundell, Tim Lillicrap, Daan Wierstra, et al. Matching networks for one shot learning. In Advances in Neural Information Processing Systems, pages 3630-3638.

[33] Zhang, J., Gao, R.X. Deep Learning-Driven Data Curation and Model Interpretation for Smart Manufacturing. Chin. J. Mech. Eng. 34, 71 (2021). https://doi.org/10.1186/s10033-021-00587-y

[34] Santoro A, Bartunov S, Botvinick M, et al. One-shot learning with memory-augmented neural networks[J]. arXiv preprint arXiv: $1605.06065,2016$

[35] Koch G, Zemel R, Salakhutdinov R. Siamese neural networks for one-shot image recognition [OL]. [2020-08-31]. http://www.cs.cmu.edu/ rsalakhu/papers/oneshot1.

[36] V Garcia and J. Bruna. Few-shot learning with graph neural networks. arXiv preprint arXiv:1711.04043, 2017

\section{Biographical notes}

Shuai Yang, born in 1986, is currently an associate professor with Chongqing Technology and Business University, Chongqing, China. He received his $\mathrm{Ph}$. D. degree from University of Ottawa, Canada. His research interests include vibration control and mechanical fault diagnosis. Tel: +86-23-62933621; E-mail: jerryyang@ctbu.edu.cn

Xu Chen, born in 1994, is currently a postgraduate student at Chongqing Technology and Business University, China. She received her Bachelor's degree in Dalian ocean university, China, in 2017. E-mail: chenxu2755473272@163.com

Yun Bai, born in 1985, is currently an associate professor with Chongqing Technology and Business University, Chongqing, China, and also is a postdoctoral researcher at University of Algarve, Portugal. His current research interests include intelligent system modeling and management. E-mail: yunbai@ctbu.edu.cn

Ziqiang Pu, he is currently a Ph.D. candidate at University of Algarve, Portugal. He received his Bachelor's degree from Chongqing Technology and Business University, China, in 2016. His research interests include mechanical fault diagnosis and intelligent systems. E-mail: a66835@ualg.pt

\section{Appendix}

none 